\title{
Aplicaciones del plasma rico en plaquetas como terapia en dermatología
}

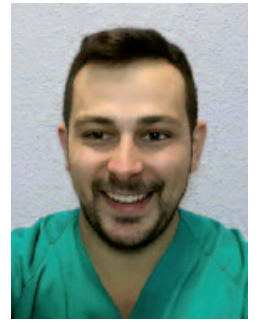

Miguel Antonio DíazMartínez Unidad de Gestión Clínica de Dermatología. Hospital Universitario San Cecilio. Granada.

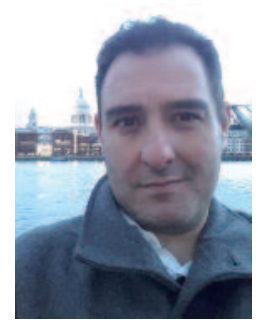

Ricardo Ruiz-Villaverde Unidad de Gestión Clínica de Dermatología. Hospital Universitario Virgen de las Nieves. Granada.

\begin{abstract}
RESUMEN
La aplicación del plasma rico en plaquetas (PRP) en diversos campos de la medicina es una terapia en pleno auge. En los últimos años, se está comunicando su aplicación de forma exponencial tanto en úlceras cutáneas crónicas como en el campo de la estética. El PRP está compuesto por distintos tipos de moléculas bioactivas que intervienen en el mecanismo de cicatrización tisular, tales como el factor de crecimiento fibroblástico de tipo 2 (FGF-2) y el factor de crecimiento derivado de las plaquetas (PDGF), implicados en la cicatrización de las úlceras, gracias a su función, que promueve la angiogénesis y la proliferación de fibroblastos dérmicos. Otro componente es el FGF-7 o factor de crecimiento epidérmico, que actúa activando la proliferación de los queratinocitos, estimulando el crecimiento del pelo.
\end{abstract}

Palabras clave: plasma rico en plaquetas, úlceras cutáneas crónicas, alopecia, factores de crecimiento.

\begin{abstract}
The application of platelet-rich plasma (PRP) to different medical fields is a rapidly expanding therapy. In the last years, its exponential application has been reported both to chronic cutaneous ulcers and to esthetics. PRP is composed of different types of bioactive molecules that take part in the tissue scarring process, such as type 2 fibroblastic growth factor (FGF-2) and platelet-derived growth factor (PDGF), involved in ulcer healing thanks to their function, which promotes angiogenesis and proliferation of dermal fibroblasts. Another component is FGF-7 or epidermal growth factor, which acts activating keratinocyte proliferation, stimulating hair growth.
\end{abstract}

Keywords: platelet-rich plasma, chronic cutaneous ulcers, alopecia, growth factors.

\section{INTRODUCCIÓN}

El plasma rico en plaquetas (PRP) es la fracción del plasma derivada de sangre autóloga que, tras ser procesada, presenta una concentración de plasma mayor a la normal. Aunque la concentración óptima de plaquetas en el PRP no está clara, se entiende que el nivel de plaquetas es de tres a cinco veces superior al nivel basal $(150000-300000 \text { plaquetas } / \mathrm{ml})^{1}$.

El método de extracción del PRP se basa en el aislamiento de plaquetas tras la centrifugación de la sangre total junto con anticoagulante. Después de aplicar la centrifugación, se observan diferentes densidades dispuestas a modo de capa, mostrando 
los diferentes componentes de la sangre, como concentrado de hematíes, fase leucocitaria y plasma. El PRP se localiza en la zona más inferior del plasma en contacto con la interfase leucocitaria. En el momento actual, no hay un método óptimo para la obtención de PRP, encontrándose en el mercado diversos kits comerciales que difieren en parámetros tales como el tiempo de centrifugación, el envase que contiene la sangre y el anticoagulante usado. Como consecuencia de ello, el PRP obtenido varía en la cantidad de plaquetas y de leucocitos y en las concentraciones de factores de crecimiento que se encuentran en él ${ }^{2}$, en los cuales residen la mayoría de las funciones responsables de su eficacia. Consecuentemente, la nomenclatura del PRP engloba las diferentes fracciones que se pueden obtener en función del método empleado: preparado rico en factores de crecimiento, PRP y factores de crecimiento, PRP y rico en leucocitos, rico en plaquetas y pobre en leucocitos.

Las plaquetas son un reservorio importante de factores de crecimiento en el organismo, interviniendo estos en la coagulación, la respuesta inmunitaria, la angiogénesis y la cicatrización de tejidos dañados. Los gránulos alfa de las plaquetas contienen diversas proteínas: factor de crecimiento derivado de las plaquetas (PDGF), factor de crecimiento transformante, interleucinas derivadas de las plaquetas, factor de angiogénesis derivado de plaquetas, factor de crecimiento endotelial vascular, factor de crecimiento epidérmico, factor de crecimiento insulinoide y fibronectina. Estas proteínas actúan llevando a cabo las funciones anteriormente citadas mediante mecanismos autocrinos, paracrinos y endocrinos ${ }^{3}$.

\section{APLICACIONES DEL PLASMA RICO EN PLAQUETAS IN VITRO}

El PRP muestra una influencia significativa en la regeneración de tejidos en los ensayos clínicos. En diversos estudios experimentales, como el de Kakudo et $a l^{4}$, en el que se comparó el uso de diferentes suplementos añadidos al medio de culti- vo - sangre total completa, PRP no activado, PRP activado, y plasma pobre en plaquetas activado añadidos al Medio de Cultivo Eagle Modificado de Dulbecco (DMEM) - , se obtuvieron resultados significativos con mayor recuento de células viables en el grupo del PRP activado, usando como activador el cloruro de calcio y la trombina autóloga. Por otro lado, se realizó otro estudio paralelo para determinar qué concentración de PRP era más efectiva al suplementar con el 0 (grupo de control), el 1, el 5, el 10 o el $20 \%$ de PRP, activando el medio DMEM. En dicho estudio, se comprobó que el mayor número de células viables se obtenía con una suplementación del DMEM al $5 \%$ en cultivos de células pluripotenciales derivadas de adipocitos y fibroblastos dérmicos humanos. De esta manera, se demostró la utilidad de suplementos de PRP en cultivos de las células componentes del estrato dérmico e hipodérmico de la piel. Asimismo, en el estudio de Rothan et al. ${ }^{5}$, se comparó la efectividad del PRP en medios de dos y tres dimensiones (2D y 3D) con medios enriquecidos con suero bovino fetal, demostrándose la mayor proliferación y diferenciación de fibroblastos dérmicos con respecto a los enriquecidos con suero bovino fetal. Los parámetros estudiados hicieron referencia a estudios de viabilidad y morfología celular, cuantificación de colágeno producido y cuantificación de actina alfa de músculo liso, lo que indicó mayor diferenciación a miofibroblastos ${ }^{5}$. En otros estudios, se ha demostrado que el PRP usado como suplemento en el medio de cultivo representaría un sustituto óptimo para el suero animal e, in vitro, se ha logrado una diferenciación hacia otros tipos celulares, como las células madre mesenquimatosas derivadas de la médula ósea a un fenotipo de fibroblastos $^{6}$.

\section{APLICACIONES DEL PLASMA RICO EN PLAQUETAS EN ÚLCERAS CUTÁNEAS}

Los fibroblastos dérmicos desempeñan un rol importante en la cicatrización mediante su proli- 
feración celular, especialización celular y formación de matriz extracelular. Como se ha expuesto antes, en situaciones simuladas, el PRP activado tiene efectos coadyuvantes en estas funciones celulares. Por otro lado, en algunos trabajos, se ha demostrado que el PRP posee actividad antimicrobiana contra Escherichia coli, Staphylococcus aureus, Candida albicans y Cryptococcus neoformans ${ }^{7}$.

Las úlceras cutáneas se corresponden con una pérdida de sustancia que afecta a la epidermis, a la dermis y, en ocasiones, a planos más profundos. En las úlceras crónicas, se observa mayor presencia de citocinas proinflamatorias que en las úlceras agudas, en un intento por llegar a la cicatrización completa. De todas las citocinas proinflamatorias estudiadas, la única comercializada para el tratamiento en la úlcera diabética es el dímero BB del PDGF.

El primer estudio de prevalencia de úlceras crónicas de las extremidades inferiores en la población española demostró una prevalencia del $0,16 \%{ }^{8}$. Las úlceras cutáneas más prevalentes en nuestro medio son las vasculares de extremidades inferiores, las del pie diabético y las úlceras por presión. Al tratamiento clásico de las úlceras cutáneas crónicas - basado en la limpieza con suero y desbridamiento de tejidos desvitalizados, curas húmedas con la utilización de distintos apósitos e hidrocoloides, y el empleo de diversas terapias compresivas, como diferentes sistemas de vendaje $^{9}$ - , se añade el uso de factores de crecimiento y otras sustancias biológicamente activas en la cicatrización de estas.

Hay diferentes estudios en la literatura médica para determinar la efectividad del PRP. En uno de los primeros ensayos clínicos aleatorizados realizados de Knighton et al..$^{10}$, en el que se comparó la efectividad del PRP frente al placebo en la curación en 32 pacientes durante ocho semanas, se obtuvieron resultados significativos en el tiempo de reepitelización de las úlceras. El grupo de Knighton usó un producto conocido como platelet-derived wound healing formula ${ }^{10}$. A partir de ese momento, se han probado los prepara- dos plaquetarios en distintas formulaciones galénicas, como preparados en solución, en gel ${ }^{11} \mathrm{o}$ en aplicación intralesional, demostrándose efectividad en el acortamiento del tiempo de cicatrización de la úlcera, así como mejoría en cuestionarios de calidad de vida y disminución del gasto sanitario ${ }^{12}$.

Estos resultados han sido ampliamente estudiados en úlceras diabéticas ${ }^{13} \mathrm{y}$, con menos profusión, en úlceras venosas e hipertensivas. En uno de los últimos estudios publicados en España, en el que se incluyen úlceras de diferente etiología no isquémica, se valora la mejoría de la superficie de la úlcera, el dolor y la calidad de vida, con muy buenos resultados, aunque cabe destacar que, en este estudio, se usa como control el propio paciente antes de iniciar el tratamiento y está limitado por el escaso número de pacientes incluidos ${ }^{14}$.

Además de la utilidad del PRP como terapia única, se han descrito trabajos de su uso con coadyuvantes como la heparina conjugada con fibrina (HCF), que actúa como una molécula con afinidad para unirse a los factores de crecimiento y realizar una liberación prolongada de dichos factores de crecimiento como el factor de crecimiento fibroblástico de tipo 2 (FGF-2) y el dímero BB del PDGF, conduciendo a un crecimiento celular mantenido in vitro y a una cicatrización más rápida que el PRP activado por calcio o el PRP unido a fibrina. El estudio de Yang et al. ${ }^{15}$ demuestra un cierre de las úlceras en menor tiempo que el PRP con HCF. Uno de los componentes principales del PRP es el FGF-2, el cual se ha demostrado que, tras administrarlo de forma exógena, mejora la regeneración del tejido conectivo y el cierre de la úlcera gracias a la estimulación de la angiogénesis ${ }^{16}$. Tras aplicar FGF-2 con HCF, se observó un mayor grosor de la epidermis y una mejoría en la diferenciación de las citoqueratinas suprabasales, que se debería a una liberación sostenida en el tiempo del FGF-217 (fig. 1).

El PRP también está siendo usado como adyuvante en el tratamiento de las úlceras con injer- 


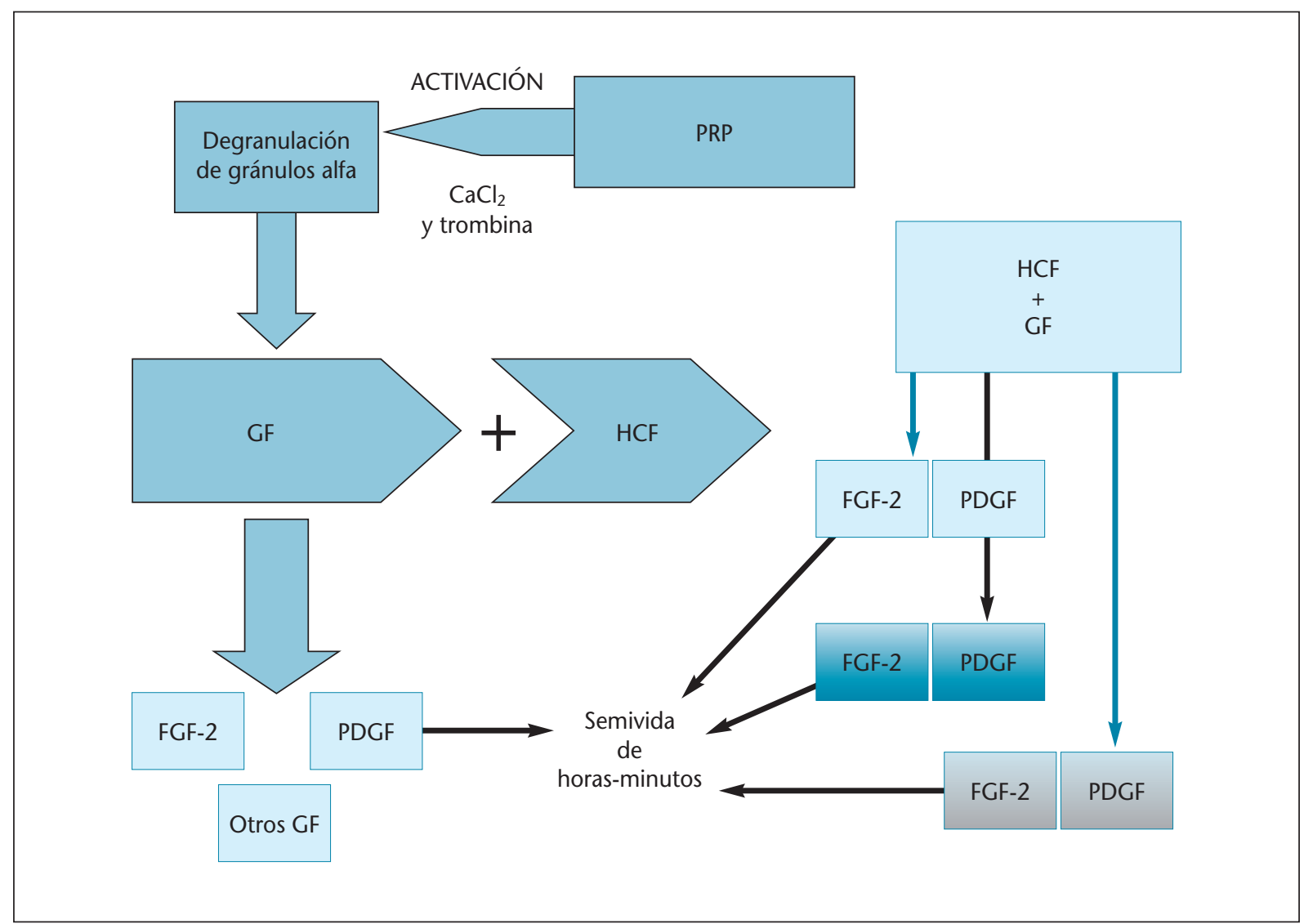

Figura 1. Esquema de la liberación de citocinas por el PRP con y sin la presencia de HCF en el PRP.

$\mathrm{CaCl}_{2}$ : cloruro cálcico; FGF-2: factor de crecimiento fibroblástico de tipo 2; GF: factores de crecimiento; HCF: heparina conjugada con fibrina; PDGF: factor de crecimiento derivado de plaquetas; PRP: plasma rico en plaquetas.

tos. Aplicado en el lecho de la úlcera en forma de gel plaquetario junto con pegamento de fibrina previamente a la aposición del injerto, se han obtenido excelentes resultados ${ }^{18}$. Por otro lado, esta actividad adhesiva se emplea en otras cirugías dermatológicas que implican la utilización de colgajos e injertos y, de esta forma, mejora la hemostasia y disminuye la reacción inflamatoria postoperatoria $^{19}$.

\section{APLICACIONES DEL PLASMA RICO EN PLAQUETAS EN LA ALOPECIA}

El pelo es una estructura compuesta por queratina y dispuesta por casi toda la superficie cutánea, que crece sobre una invaginación epidérmica cono- cida como folículo pilosebáceo. En la base del folículo pilosebáceo, se encuentran las células de la papila dérmica, un componente del mesénquima que desempeña un papel crucial en el desarrollo del folículo, puesto que controla su regeneración cíclica ${ }^{20}$ en tres fases de crecimiento: anágeno (fase de crecimiento), catágeno (fase involutiva) y telógeno (fase quiescente).

El crecimiento del pelo y su ciclo están regulados por señales dérmicas y epidérmicas que reciben las células de la papila dérmica por medio de una vía de señalización Wnt (vía de señalización canónica utilizada en el crecimiento embrionario), cuyo resultado es una acumulación de $\beta$-catenina en el citoplasma y migración al núcleo 
para unirse a unas proteínas activadoras de la familia TCF4, cuyo objetivo es la proliferación celular y, así, el crecimiento del pelo ${ }^{21}$. Por otro lado, hay otras vías de señalización que intervienen en el crecimiento del pelo, como las quinasas, por señalización extracelular que induce la proliferación celular, y la vía de señalización Akt1, que promueve la supervivencia celular evitando la apoptosis.

En el estudio de Li et al. ${ }^{22}$, tras aplicar PRP en un cultivo de células de la papila dérmica, se demostró un aumento de la proliferación determinado por el ensayo de incorporación de timidina tritiada. Además, se observó un aumento de las pERK y las pAkt. Por otro lado, en el grupo del cultivo celular suplementado con un $5 \%$ de PRP, se observó una mayor expresión de la proteína antiapoptótica bcl-2 y un incremento de la cantidad de factor de crecimiento epidérmico FGF7 en el medio de cultivo (fig. 2). Asimismo, tam- bién se demostró un aumento de actividad de la $\beta$-catenina ${ }^{22}$. En este trabajo, además, se realizó un estudio in vivo en el que, tras la aplicación de PRP en el dorso de ratones en fase de telógeno, se demostró su capacidad para acelerar el paso a anágeno.

La aplicación del PRP en humanos ha sido estudiada en pacientes con alopecia areata en un ensayo clínico frente a placebo y acetónido de triamcinolona, con mejores resultados para la aplicación intralesional de PRP valorado mediante el aumento de la expresión del antígeno Ki-67 como indicador de proliferación celular. Su efectividad clínica ha permitido a los autores presuponer efectos antiinflamatorios análogos a los descritos en estudios previos ${ }^{23}$. Otras indicaciones clínicas que precisan estudios más consolidados incluyen el uso de PRP en cirugía para trasplante capilar, obteniéndose mayor rendimiento de los microinjer-

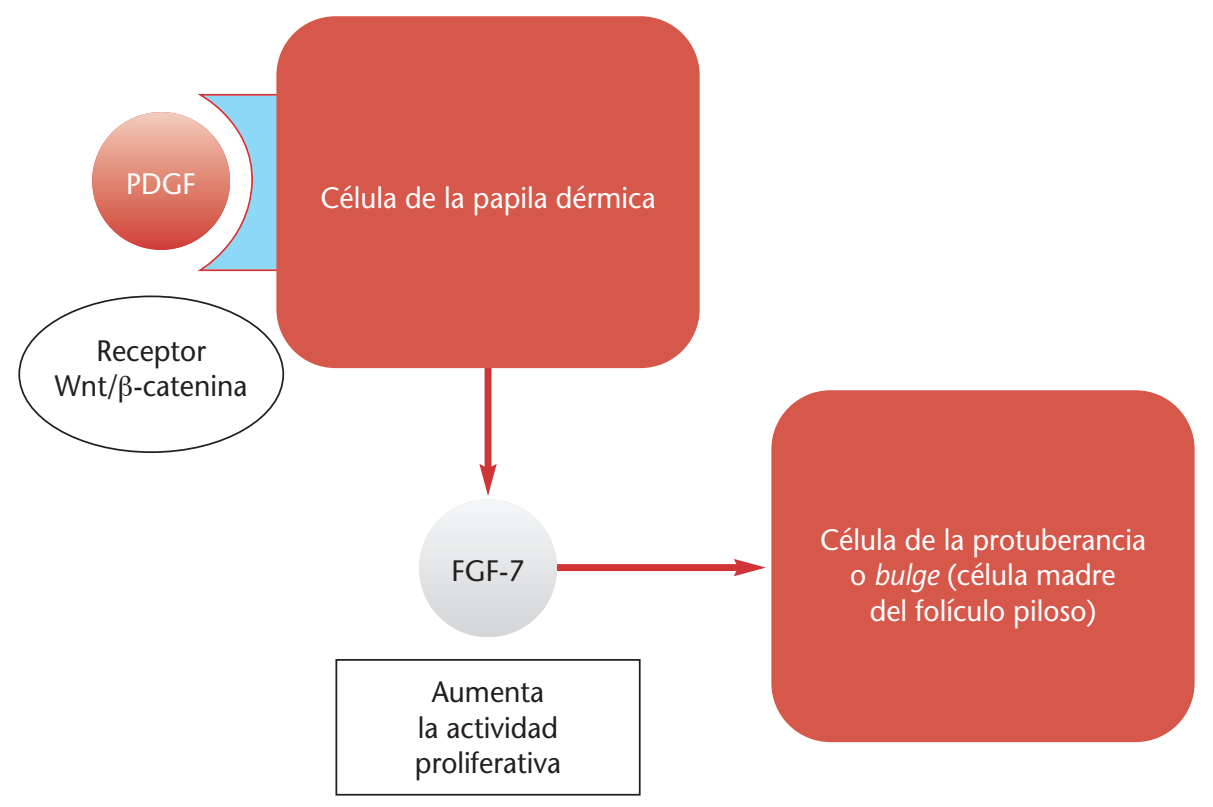

Figura 2. Interacción entre una célula de la papila dérmica y una célula de la protuberancia (bulge). Una de las vías de Wnt/ $\beta$ catenina libera de la célula de la papila FGF-7, que activa la proliferación de las células madre del folículo pilosebáceo.

FGF-7: factor de crecimiento fibroblástico de tipo 7 o factor de crecimiento epidérmico; PDGF: factor de crecimiento derivado de plaquetas. 
$\operatorname{tos}^{24}$ cuando su aplicación se espacia respecto a la propia cirugía.

\section{APLICACIONES DEL PLASMA RICO EN PLAQUETAS EN ESTÉTICA FACIAL}

La utilidad del PRP se ha evaluado, principalmente, en las cicatrices de acné, mostrando buenos resultados frente a otras modalidades de tratamiento. En esta ocasión, se ha basado en la realización de microincisiones cutáneas con posterior aplicación del PRP en forma de gel o inyección subcutánea. La mejoría de la apariencia de las cicatrices atróficas se basaría en una reordenación del colágeno gracias a los factores de crecimiento presentes en el PRP. Además, su aplicación en el rejuvenecimiento facial provoca mejoría en la firmeza y elasticidad de la piel, tal y como Yuksel et al. ${ }^{25}$ han demostrado recientemente.

\section{CONCLUSIONES}

La aplicación del PRP es un tratamiento en expansión, seguro, eficaz y que actúa, principalmente, a nivel local cutáneo, pero que también se empieza a utilizar en múltiples campos de la medicina como la traumatología y la rehabilitación.

En el campo de la dermatología, su uso en úlceras crónicas parece estar en fase de afianzamiento, mientras que sus aplicaciones estéticas crecen de forma exponencial. En esta revisión, se ha pretendido fundamentar los conocimientos fisiopatológicos básicos del PRP para comprender mejor su aplicación en la terapéutica dermatológica. No obstante y a pesar de los prometedores resultados en las úlceras, la alopecia y el campo de la estética, la mayoría de estudios mencionados incluyen un limitado número de pacientes, lo que hace, en ocasiones, difícil la extrapolación para su uso en la práctica clínica habitual. Son precisos, pues, estudios con mayor casuística para poder reafirmar las bondades en eficacia y efectividad del PRP, sin olvidar los criterios de seguridad que en la actualidad se exigen a estos productos.

\section{BIBLIOGRAFÍA}

1. Wroblewski AP, Melia HJ, Wright VJ. Application of plateletrich plasma to enhance tissue repair. Oper Tech Orthop. 2010;20:98-105.

2. Kesy L, Kopczy ski P, Baszckuk A, Kopczy ski Z. Methods of preparation of the platelet-rich plasma used in medicine as an accelerator of tissue regeneration. Pol Merkur Lekarski. 2014;36(214):283-6.

3. Lubkowska A, Dolegowska B, Banfi G. Growth factor content in PRP and their applicability in medicine. J Biol Regul Homeost Agents. 2012;26(2 Suppl 1):3S-22S.

4. Kakudo N, Minakata T, Mitsui T, Kushida S, Notodihardjo FZ, Kusumoto K. Proliferation-promoting effect of plateletrich plasma on human adipose-derived stem cells and human dermal fibroblasts. Plast Reconstr Surg. 2008;122(5):135260.

5. Rothan HA, Djordjevic I, Bahrani H, Paydar M, Ibrahim F, Abd Rahmanh N, et al. Three-dimensional culture environment increases the efficacy of platelet rich plasma releasate in prompting skin fibroblast differentiation and extracellular matrix formation. Int J Med Sci. 2014;11(10):1029-38.

6. Formigli L, Benvenuti S, Mercatelli R, Quercioli F, Tani A, Mirabella $C$, et al. Dermal matrix scaffold engineered with adult mesenchymal stem cells and platelet-rich plasma as a potential tool for tissue repair and regeneration. J Tissue Eng Regen Med. 2012;6(2):125-34.

7. Bielecki TM, Gazdzik TS, Arendt J, Szczepanski T, Król W, Wielkoszynski T. Antibacterial effect of autologous platelet gel enriched with growth factors and other active substances: an in vitro study. J Bone Joint Surg Br. 2007;89(3):417-20.

8. Torra Bou JE, Soldevilla Agreda JJ, Rueda López J, Verdú Soriano J, Roche Rebollo E, Arboix Perejamo M, et al. Primer estudio nacional de prevalencia de úlceras de pierna en España. Estudio GNEAUPP-UIFC-Smith \& Nephew 2002-2003. Epidemiología de las úlceras venosas, arteriales, mixtas y de pie diabético. Gerokomos. 2004;15(4):230-47.

9. Moreno-Giménez JC, Galán-Gutiérrez M, Jiménez-Puya R. Tratamiento de las úlceras crónicas. Actas Dermosifiliogr. 2005;96(3):133-46.

10. Knighton DR, Ciresi K, Fiegel VD, Schumerth S, Butler E, Cerra F. Stimulation of repair in chronic, nonhealing, cutaneous ulcers using platelet-derived wound healing formula. Surg Gynecol Obstet. 1990;170(1):56-60.

11. Kazakos K, Lyras DN, Venettas D, Tilkeridis K, Tryfonidis M. The use of autologous PRP gel as an aid in the management of acute trauma wounds. Injury. 2009;40(8):801-5.

12. Carter MJ, Fylling CP, Parnell LK. Use of platelet rich plasma gel on wound healing: a systematic review and meta-analysis. Eplasty. 2011;11:e38.

13. Driver VR, Hanft J, Fylling CP, Beriou JM; Autologel Diabetic Foot Ulcer Study Group. A prospective, randomized, controlled trial of autologous platelet-rich plasma gel for the treatment of diabetic foot ulcers. Ostomy Wound Manage. 2006;52(6):68-87.

14. Salazar-Álvarez AE, Riera-del-Moral LF, García-Arranz M, Álvarez-García J, Concepción-Rodríguez NA, Riera-de-Cubas L. Use of platelet-rich plasma in the healing of chronic ulcers of the lower extremity. Actas Dermosifiliogr. 2014;105(6):597604.

15. Yang HS, Shin J, Bhang SH, Shin JY, Park J, Im Gl, et al. Enhanced skin wound healing by a sustained release of growth 
factors contained in platelet-rich plasma. Exp Mol Med. 2011; 43(11):622-9.

16. McGee GS, Davidson JM, Buckley A, Sommer A, Woodward SC, Aquino AM, et al. Recombinant basic fibroblast growth factor accelerates wound healing. J Surg Res. 1988;45(1):14553.

17. Bhang SH, Sun AY, Yang HS, Rhim T, Kim DI, Kim BS. Skin regeneration with fibroblast growth factor 2 released from heparin-conjugated fibrin. Biotechnol Lett. 2011;33(4):845-51.

18. Chen TM, Tsai JC, Burnouf T. A novel technique combining platelet gel, skin graft, and fibrin glue for healing recalcitrant lower extremity ulcers. Dermatol Surg. 2010;36(4):453-60.

19. Findikcioglu F, Findikcioglu K, Yavuzer R, Lortlar N, Atabay K. Effect of preoperative subcutaneous platelet-rich plasma and fibrin glue application on skin flap survival. Aesthetic Plast Surg. 2012;36(5):1246-53.

20. Botchkarev VA, Paus R. Molecular biology of hair morphogenesis: development and cycling. J Exp Zool B Mol Dev Evol. 2003;298(1):164-80.
21. Xiong Y, Liu Y, Song Z, Hao F, Yang X. Identification of Wnt/Bcatenin signaling pathway in dermal papilla cells of human scalp hair follicles: TCF4 regulates the proliferation and secretory activity of dermal papilla cell. J Dermatol. 2014;41(1): 84-91.

22. Li ZJ, Choi HI, Choi DK, Sohn KC, Im M, Seo YJ, et al. Autologous platelet-rich plasma: a potential therapeutic tool for promoting hair growth. Dermatol Surg. 2012;38(7 Pt 1):1040-6.

23. El-Sharkawy H, Kantarci A, Deady J, Hasturk H, Liu H, Alshahat $\mathrm{M}$, et al. Platelet-rich plasma: growth factors and pro- and anti-inflammatory properties. J Periodontol. 2007;78(4): 661-9.

24. Uebel CO, da Silva JB, Cantarelli D, Martins P. The role of platelet plasma growth factors in male pattern baldness surgery. Plast Reconstr Surg. 2006;118(6):1458-66.

25. Yuksel EP, Sahin G, Aydin F, Senturk N, Turanil AY. Evaluation of effects of platelet-rich plasma on human facial skin. J Cosmet Laser Ther. 2014;16(5):206-8. 